\title{
The Relationship between Cardiorespiratory Fitness and Allostatic Load Claudia Traunmüller $^{{ }^{*}}$, Peter Hofmann ${ }^{2}$, Kerstin Gaisbachgrabner $^{1}$, Alexander Müller ${ }^{2}$, Karoline Vrecko $^{3}$, Andreas \\ Rössler ${ }^{4}$ and Andreas Schwerdtfeger ${ }^{1}$ \\ ${ }^{1}$ Department of Psychology, Health Psychology Unit, University of Graz, Graz, Austria \\ ${ }^{2}$ Institute of Sports Science, Exercise Physiology, Training \& Training Therapy Research Group, University of Graz, Graz, Austria \\ ${ }^{3}$ Institute of Physiological Chemistry, Medical University of Graz, Graz, Austria \\ ${ }^{4}$ Institute of Physiology, Medical University of Graz, Graz, Austria
}

\begin{abstract}
Background: There is evidence that chronic stress/burnout have a negative impact on physical and mental health. Annually global burden of stress related disorders amounts to 300 billion dollars. Conversely, physical fitness seems to have a significant influence on the development of a large number of mental and physical disorders and on reduction of physical stress. The aim of this study was to examine stress related cumulative physiological burden in relation to cardiorespiratory fitness level.
\end{abstract}

Methods: A sample of 86 healthy males aged between 28-60 years from an international company took part in the study. In addition to questionnaires, an indicator of cumulative physiological burden (allostatic load) was calculated including systolic and diastolic blood pressure, waist to hip ratio and 24-hour overnight urinary epinephrine and norepinephrine, cortisol and HRV variables (SDNN, rMSSD and LF/HF). All individuals underwent additionally a maximal incremental exercise test on an electronically braked cycle ergometer.

Results: All participants showed high working stress, measured by effort/reward ration. High allostatic load was significantly associated with low cardiorespiratory fitness. Individuals with higher cardiovascular fitness showed significantly lower WHR, HR, DBP and significantly higher SDNN and rMSSD compared to participants with lower cardiorespiratory fitness.

Conclusion: Signs of work-stress related physiological burden were significantly related to cardiorespiratory fitness. Significant reduction of physiological burden could be observed if people achieved more than $120 \%$ of age related cardiovascular fitness but not if participants felt below this threshold.

\section{Introduction}

There is evidence that chronic stress has a negative impact on health. The global burden of stress related disorders respectively burnout through decreased productivity, retention, absenteeism, and compensation costs in excess of 300 billion dollars annually worldwide [1]. It is expected that the World Health Organization (WHO) cite stress/burnout as a global pandemic within the next decade [2]. Disorders like obesity, metabolic syndrome, type 2 diabetes, heart diseases, allergy, anxiety, depression, fatigue syndrome and burnout are often associated with dysfunctions of the stress axes [3]. The probability of such dysfunctions can be affected by the individual's biological vulnerability, learned patterns of coping and the number and intensity of high stress exposure [4]. It might be assumed that prospective health will depend strongly on the adaptive or maladaptive adjustments to high demanding and rapidly changing environments.

The biological response to stress is mirrored by a cascade of changes in different physiological systems (e.g., nervous, cardiovascular, endocrine and immune systems) [5]. These stress systems follow a circadian activity and serve to adapt the organism to challenging circumstances $[3,6]$. For sustained well-being and the ability of good performance at work a coordinated and functional stress system, which is able to respond adequately to stress-situations seems mandatory. An adequate stress response can be characterized by a rather short-lived response in the face of threat and a rapid recovery thereafter. Contrary, stress systems which respond inappropriate to stress may be detrimental for health [7].

\section{Publication History:}

Received: September 09, 2016

Accepted: January 31, 2017

Published: February 02, 2017

\section{Keywords:}

Autonomic nervous system, Burnout, Cardiorespiratory fitness, Stress
This development ends in a lack of feeling of existence as well as meaning of life, leading to a feeling of inner emptiness and existential despair. According to Maslach et.al. [5], three main dimensions of these symptoms can be summed up as follows: (a) emotional exhaustion (e.g. fatigue and meaninglessness), (b) depersonalisation (e.g. setting aside personal interests as well as interactions with the environment) and (c) inefficacy, reduced personal accomplishment which results in an initially only feared but increasingly real-noncompliance of tasks and duties.

In previous studies, efforts have been undertaken to examine the association between exposure to workplace stress and health-related consequences [8-11]. One key model of work stress is the EffortReward Imbalance (ERI) [12] model focusing on the concept of social reciprocity, meaning a balanced ratio between effort and reward [13]. Violation of this reciprocity may lead to negative emotions and sustained stress. A meta-analysis indicated a 50\% higher risk for heart diseases among employees with work stress as evidenced by lower effort/reward-ratio [14]. Of note, this finding could be attributed to "Corresponding Author: Dr. Claudia Traunmüller, Health Psychology Unit, Department of Psychology, University of Graz,Universitaetsplatz 2/III, $8010 \mathrm{Graz}$, Austria, Tel: +43 316-380 4950; E-mail: claudia.traunmueller@uni-graz.at

Citation: Traunmüller C, Hofmann P, Gaisbachgrabner K, Müller A, Vrecko K, et al. (2017) The Relationship between Cardiorespiratory Fitness and Allostatic Load. Int J Phys Ther Rehab 3: 127. doi: https://doi.org/10.15344/2455$7498 / 2017 / 127$

Copyright: @ 2017 Traunmüller et al. This is an open-access article distributed under the terms of the Creative Commons Attribution License, which permits unrestricted use, distribution, and reproduction in any medium, provided the original author and source are credited. 
two pathways: a possible indirect effect via health risk behaviors like smoking, sedentarism, malnutrition, and alcohol consumption [15] as well as direct effects on neuro-endocrine stress responses [16] that could result in physiological burden [17]. In this context, two physiological systems are commonly distinguished in the literature: The sympathetic-adrenergic-medullary (SAM) axis, releasing catecholamines, and the hypothalamic-pituitary adrenal (HPA) axis by secretion of cortisol [18]. The activation of the sympathetic system can be assessed in different ways: Measurement of catecholamine levels (epinephrine and norepinephrine) using integrated methods like urine collections over night or over 24 hours as well as measurement methods that may capture the autonomic nervous system function more generally by assessing sympathetic and parasympathetic activity To reflect the balance between sympathetic and parasympathetic activity it is common to measure heart rate (HR) and/or heart rate variability (HRV). HRV is widely discussed as a marker of autonomic influences on the heart [7]. The HPA axis plays a key role during stress. Its activation is accompanied by an increase in cortisol release in order to provide energy to the body needed to handle acute stress situations. The diurnal rhythm in cortisol release and its various elements like the increase (50-60\%) of cortisol concentration within the first $30-45$ minutes after awakening - the so called cortisol awakening response (CAR) - are viewed as essential indicators of HPA axis functioning [19]. There is evidence for an association between work stress and increased levels of catecholamines, lower levels of HRV and greater CAR [7]. The authors concluded that work stress is accompanied by elevated stress response in terms of sympatho-adrenal and HPA axis biomarkers. The costs of chronic exposure to repeated or chronic stress on the organism can be defined as Allostatic load (AL) [20].

\section{Concept of allostatic load (AL)}

Health can be seen as the ability of an individual to either adapt or effectively respond to rapidly changing environments [4]. The allostatic load model has been developed to assess physiological dysregulations resulting from prolonged and constantly recurring stress. Healthy individuals are basically able to maintain physical stability through constant adaptation and matching their internal milieu to environmental demands. Typically this process (so-called allostasis [21]) does not impose health burden with respect to shortterm stressors [4]. During the allostatic process and after responding to a stressful situation, the organism strives for returning to normal values - which depends on the time to recover. In contrast, if allostatic responses are repeatedly activated by the nature, number, and persistence of the stressors, this may lead to allostatic load (AL), the so-called wear and tear of the body [20].

There is evidence for individual differences in the stress response [22]. Individual differences in physiological responding to stress call for an aggregated measure of physiological load on the organism in order to get an overview of the extent of the current physiological state of a person [23]. As an indicator of biological dysregulations in different physiological systems, a so-called allostatic load index (ALI) can be calculated to index cumulative physiological burden. Previous research examined the relationship between physical activity and $\mathrm{AL}$ in Mexican Americans [24]. Results showed significantly lower AL in high active participants compared to sedentary individuals. This goes in line with results from other studies showing a positive effect of physical activity on AL $[25,26]$.

\section{Cardiorespiratory fitness}

Physical activity and exercise has an impact on many aspects of health [27]. In this context it is necessary however to distinguish between the terms physical activity, physical exercise and physical fitness, which describe different concepts. They are often confused with one another, and the terms are sometimes used interchangeably. Physical activity is defined as any bodily movement produced by skeletal muscles that results in energy expenditure measured in kilojoules. Physical activity in daily life can be categorized into occupational, sports, conditioning, household, or other activities. Exercise is a subset of physical activity that is planned, structured, and repetitive and has as a final or an intermediate objective the improvement or maintenance of physical fitness. Physical fitness is a set of attributes that are either health- or skill-related. It can be measured with specific tests, usually incremental exercise tests to measure cardiorespiratory fitness (CRF) [28]. Comparably high levels of physical fitness seem to have a significant influence on the development of a large number of mental and physical disorders. The positive impact of physical activity on health is often seen as a protective factor against the development of mental diseases like depression or anxiety symptoms [29-31] as well as against the physical consequences of intensive stress, like cardiovascular diseases [32,11]. Results of a meta-analysis confirmed a moderating effect of physical activity on physiological reactivity to mental stress [33]. Moreover, a physically active lifestyle is suggested to reduce stress [27]. Taken together, these results may imply that physical fitness could decrease the physiological burden of stress.

There is evidence concerning the impact of aerobic cardiovascular exercise on burnout and perceived stress [34, 35]. Results suggest a large burnout-reducing effect as well as an amelioration of perceived stress. Additionally, research found significant associations between frequency of exercise and perceived stress, well-being and burnout [36]. Importantly, these studies are based on self-report measures of stress and physical activity and no measures of physical fitness have been assessed in most studies. Recently, it was reported that physical activity, CRF, and body composition were negatively associated with levels of stress and recovery on workdays in a cross-sectional study involving 81 healthy males [37]. Overall, $27.5 \%$ of the variance of total stress on workdays was accounted for by physical activity, CRF, and body composition. Body fat percentage and body mass index were negatively associated with night-time recovery whereas for CRF the association was positive. Hence, analysing CRF in relation to workrelated stress and its psychophysiological concomitants seems to be worthwhile.

To our knowledge, the impact of CRF on cumulative physiological burden symptoms has not been systematically studied by now and the assessment of CRF using a maximal incremental exercise test is lacking in this respect. CRF was shown to be an objective measure of habitual physical activity and a useful diagnostic and prognostic health indicator [38]. Although compelling evidence suggests that CRF is a strong and independent predictor of all-cause and cardiovascular disease mortality, the importance of CRF is often overlooked from a clinical perspective compared with other risk factors [39].

Contrary to previous study designs, where differences in physiological reactivity were measured during an acute stressinduction (short time), in this study we focused on an average of physiological burden symptoms, measured over 24 hours, twice a week. Hence, the generalizability to everyday life physiological burden could be ensured. Of note, the dose-response relationship between CRF and AL it is not yet known. Thus, the aim of this study was to explore the relationship between cumulative physiological burden and CRF within employees working in a high demanding 
Citation: Paletta A, Suchar G, Hübler T, Juschitz D, Mayer K, et al. (2017) Time Experience of Burnout Patients: A Qualitative Diary Analysis. Int J Phys Ther Rehab 3: 126. doi: https://doi.org/10.15344/2455-7498/2017/126

Page 3 of 7

environment. It was expected that high CRF would be related to lower signs of physiological burden respectively to lower AL.

\section{Methods}

Beside the question of satisfaction with their body, patients were also tested on how differentiated they are able to feel their body? It is argued in specialist literature that patients who suffer from psychological disturbances experience their body less differentiated than healthy people [12]. Furthermore, it can be said, that people who do not "feel" their own body and their needs are highly endangered of suffering from burnout, as they neglect the symptoms and warning signs their body sends out [16,17]. To "feel" one's body, a person has to be able to differentiate his or her body. Müller [14] argues, that a high level of differentiation leads to a stable integration of the body image into one's mind and therefore also leads to a stable concept of oneself, which is vital to avoid Burnout and other psychological malfunctions. To be able to focus on areas of dissatisfaction goes hand in hand with not feeling dissatisfied with the body as a whole, only due to a malfunction of one body part, thus stabling the concept of oneself. Once a person is able to distinguish that an impairment of one body area does not lead to a breakdown of the entire system, but rather is something that can be corrected if focused on, the way is pathed to a healthier self

So far, the experience of time was described from a phenomenological point of view and shall now be validated empirically. Besides the inquiry of patterns in personal experience of time of burnout patients, it shall also be analysed how stable these patterns are and in how far they are accessible to interventions and measures in rehab clinics.

\section{Methods}

\section{Study sample}

The study sample comprised of 98 participants, 94 males and 4 females. Because women were severely under represented in the study group they were excluded from further analyses. Exclusion criteria were: having had a traumatic event during the past six months, cardiovascular diseases (e.g., myocardial infarction and stroke), and current or a history of psychiatric illness. Participants with missing values on physiological variables were also excluded from the analysis. Four participants were excluded due to the intake of prescribed drugs for high blood pressure treatment and additional four participants due to high scoring on the short version of the Mini-DIPS interview [40] to avoid possible confounding with existing mental disorders. Consequently, the final sample size was $\mathrm{N}=86$ healthy male participants. The mean age was 41.27 years $(\mathrm{SD}=7.13$; range: $28-60)$ and participants worked on average 46.06 hours per week ( $\mathrm{SD}=5.64$; range: 38.5 - 60). Demographic and anthropometric data are shown in Table 1.

\section{Recruiting process}

Participants were recruited within an international company, which has different subsidiaries all over the world. The site, where this study was conducted, is employing a total of around 1.866 persons; 1.534 of them are own personnel and 332 are hired employees. In this project we focused on individuals in working contexts characterized by high demands. In sum, 347 individuals were eligible for participating. In a first step, all individuals on project manager level were contacted and informed about the study $(\mathrm{N}=140) ; 120$ persons responded by

\begin{tabular}{|c|c|c|}
\hline & Mean & SD \\
\hline Age & 41.16 & 7.03 \\
\hline Body mass (kg) & 84.40 & 10.80 \\
\hline Height $(\mathrm{cm})$ & 180.40 & 6.70 \\
\hline Waist-to-hip ratio & .92 & .10 \\
\hline Working Hours & 45.73 & 5.25 \\
\hline \multirow[t]{2}{*}{ Regular sporting activity } & yes & no \\
\hline & 57 & 29 \\
\hline \multirow[t]{2}{*}{ Smoker } & yes & no \\
\hline & 11 & 75 \\
\hline \multicolumn{3}{|l|}{ Education: } \\
\hline Compulsory school & \multicolumn{2}{|c|}{-} \\
\hline Secondary modern school & \multicolumn{2}{|c|}{3} \\
\hline College & \multicolumn{2}{|c|}{3} \\
\hline Higher School Certificate & \multicolumn{2}{|c|}{20} \\
\hline University degree & \multicolumn{2}{|c|}{60} \\
\hline \multicolumn{3}{|l|}{ Family Status: } \\
\hline living alone & \multicolumn{2}{|c|}{4} \\
\hline in a relationship & \multicolumn{2}{|c|}{34} \\
\hline married & \multicolumn{2}{|c|}{48} \\
\hline
\end{tabular}

Table 1: Demographic and antropometric data $(n=86)$.

e-mail and showed interest in the study. In a second step, an informal meeting was organized during whichthe study was described and detailed information was given. Confidentiality, anonymity and the opportunity to withdraw from the study without any negative repercussions was assured. Informed consent form was signed by 98 employees. A precise timetable for collecting the data was scheduled. To ensure that the recorded data were indicative of a typical workweek, attention was paid on the planned holidays or business trips of the participants. Accordingly, as a prerequisite it was assured that participantswere working at least for four weeks without interruption before data collection. The study was approved by the ethics committee of the local University.

\section{Variables and Procedure}

To enhance reliability of the data, all physiological variables were assessed twice within a week (except for Waist to hip ratio and CRF). To analyze reliability of the measurements, intraclass correlation coefficients for absolute agreement (ICCs) were calculated. Generally, ICCs ranged between .77 and .92, thus justifying the calculation of an aggregated score for each variable. ICCs of Cortisol ranged between .45 and .61 . Therefore, this variablewas not aggregated to one score.

\section{Work-Stress}

Work-stress was assessed by the Effort-Reward-Imbalance (ERI) [12] questionnaire. The effort-reward-model of stress has proven useful in predicting stress-related health complications[41]. ERI measures three psychometric scales, effort ( 3 items), reward (7 items) and over commitment ( 6 items) at job and provides an imbalance score between effort and reward by calculating the ratio between these two scales. Items are rated on 4-point Likert scales, ranging from "bad" to "very good". Scores for each scale are aggregated into a sum score, ranging from 3 to 12 for the subscale effort, 7 to 28 for reward and 6 to 
Citation: Paletta A, Suchar G, Hübler T, Juschitz D, Mayer K, et al. (2017) Time Experience of Burnout Patients: A Qualitative Diary Analysis. Int J Phys Ther Rehab 3: 126. doi: https://doi.org/10.15344/2455-7498/2017/126

Page 4 of 7

24 for over commitment. Higher scores are indicative of more stress (effort) due to perceived demands and higher levels of reward and over commitment at work [41]. It is postulated that the imbalance between effort and reward is what matters most with regard to negative health outcome and well-being. Values beyond 1.0 indicate relatively higher effort relative to lowerperceived reward, whereas values close to zero indicate less stress, meaning that perceived reward outweigh expended effort [41]. Cronbach`s alpha was .593 for effort, .798 for reward and .461 for over commitment in this study sample, indicating, with the exception of the subscale effort, rather lowreliabilities, thus calling for caution when interpreting the ERI results. The psychological tests were handed over to the participants asking them to fill out the forms and return them within one week.

\section{Allostatic load}

AL was calculated to summarize physiological activity across the regulatory systems, thus indicating cumulative physiological load. Five biological markers were used to calculatean AL_basic score: systolic and diastolic blood pressure, waist to hip ratio, and 24-hour overnight urinary epinephrine and norepinephrine. Furthermore there is evidence that work stress is highly associated with reduced HRV and reduced parasympathetic activity (rMSSD) [42]. Including HRV variablesinto the AL score has been considered usefulbecause reduced parasympathetic activity has been found in individuals affected by burnout symptoms [43]. Consequently, in this study cortisol, HR and HRV parameters (SDNN, rMSSD and LF/HF) were additionally included in an extended versionof the AL, which comprised ten biomarkers altogether.In accordance with previous research percentiles were calculated and summarized to quantify a cumulative index of allostatic load (ALI)[44]. This ALI is defined as highest 75th percentiles of each parameter (except cortisol, SDNN and rMSSD, for which the lowest 25th percentiles signified highest risk). According to previously publishedevidencereduced cortisol increase in the morning within the first 30 minutes after awakening wasdefined as a risk factor[45]. In sum, ALIsignalsthe total number of dysregulated biomarkers and could range from 0 to 5 for the "classical", and 0-10 for the "extended" AL index. Higher scores reflect greater cumulative physiological burden [46]

\section{Measurement of catecholamines and cortisol}

Participants were precisely informed how to collect saliva and the 24 hours urine samples. For the saliva samples Salivettes (Sarstedt, Nümbrecht, Germany) were used, which were labeled with the code of the participant and the date and time the samples had to be taken. The cortisol awakening response (CAR)was assessed as an essential indicator of HPA axis functioning. Participants were instructed to strictly follow the time schedule for saliva sampling. First sample collection startedimmediately upon awakeningand $+15,+30$ and +45 minutes thereafter. To obtain valid data it was important that participants completed sampling before breakfast. To avoid contamination of saliva with food or blood they were asked to not brush their teeth before data collection was finished. To increase compliance, participants were supported in developing an exact sampling schedule.According to the guidelines for assessment of the CAR [47], ECG monitoring was additionally used to validate the awakening time of the participants.

Salivary free cortisol was analyzed by using a commercial chemiluminescence immunoassay (LIA) (IBL Hamburg, Germany). To reduce error variance caused by imprecision of the intra-assay, all samples of one participant were analyzed in the same run.
The same labeling was used for the urine containers. 24 hours urine collection containers were filled with $10 \mathrm{ml} 25 \%$ hydrochloric acid $(\mathrm{HCl})$. Participants collected urine from8:00 a.m. until next day 8:00 a.m. and were instructed to sample also the first morning urine. Saliva and urine samples were taken immediately to the laboratory, the amount of urine was recorded and aliquots frozen at $-25^{\circ} \mathrm{C}$ until biochemical analysis took place.

Determination of the catecholamines norepinephrine and epinephrine was performed by high-performance liquid chromatography (HPLC) with electrochemical detection, according the extraction procedure suggested by Chromsystems (Munich, Germany).

\section{ECG-monitoring}

ECG-electrodes were attached by the experimenter and continuous $\mathrm{RR}$ intervalswere recorded by Medilog ${ }^{\circledR} \mathrm{AR} 12$ plus recorders (Medilog'AR12plus, Schiller, Switzerland) with a sampling rate of $8000 \mathrm{~Hz}$.Artifact handling of beat-to-beat HR-values was done semi-automatically by medilog ${ }^{\oplus}$ ADAPT $^{\text {tin }}$ software (medilog ADAPT $^{\text {ma }}$, Schiller, Switzerland). Single artifacts were replaced by linear interpolation and their appearance was recorded. HRV was assessed following the standard criteria of the Task Force of the European Society of Cardiology and the North American Society of Pacing and Electrophysiology [48]. Based on the evidence that psychological stress produces significant changes in sympathovagal activity [49], two of the time domain measures, standard deviation of the normal tonormal interval (SDNN) and square root of the mean squared differences of successive RR intervals (rMSSD), as well as the ratio of two frequency domain measures, low frequency component (LF), 0.04-0.15 Hz, and high frequency component (HF), 0.15-0.40 $\mathrm{Hz}$, using Fast Fourier Transformation, were analyzed in this study. RMSSD and HF are most sensitive to parasympathetic (i.e., vagal) diefference and the ratio LF/HF indicates the relative balance between sympathetic and parasympathetic system[50].

\section{Measurement of blood pressure and waist-to-hip ratio}

Blood pressure was calculated as the average of the first and the last 180 s (total of 320s) of a 10 minute continuous blood pressure measurement in a seated position. Blood pressure was measured with Task Force ${ }^{\oplus}$ Monitor V2.2 (CN systems, medical technology, Austria).

Waist-to-hip ratio (WHR) was measured on the first measurement day by the experimenter. According to the WHO abdominal obesity is defined as a waist-hip ratio above .90 for males and above .85 for females.

\section{Cardiorespiratory fitness}

All participants underwent a maximal incremental exercise test at an electronically braked cycle ergometer (MonarkErgomedic 389E, Sweden). After a careful anamnesis by a physician participants were instrumented, seated on the cycle ergometer and measures were started. Data were obtained for quiet sitting on the cycle ergometer, warm up, during incremental exercise and during active and passive recovery. All exercise tests started at $40 \mathrm{~W}$ and workload was increased by 15 or $20 \mathrm{~W}$ per min according to expected maximal performance in order to reach maximal workload within $15 \mathrm{~min}$. After termination of maximal exercise workload was decreased to 40 $\mathrm{W}$ in all individuals for $3 \mathrm{~min}$ of active recovery and measures were continued for additional $3 \mathrm{~min}$ of passive sitting on the ergometer 
Citation: Paletta A, Suchar G, Hübler T, Juschitz D, Mayer K, et al. (2017) Time Experience of Burnout Patients: A Qualitative Diary Analysis. Int J Phys Ther Rehab 3: 126. doi: https://doi.org/10.15344/2455-7498/2017/126

Page 5 of 7

before stopping the test. Throughout the test a 12-lead ECG (ZAN 800 EKG, Germany) was obtained and monitored by the study physician. Additionally, heart rate (HR) was measured continuously (S810i, Polar Electro, Finland) and data were stored in $5 \mathrm{~s}$ intervals for further analysis. A second HR monitor was used to obtain beat-tobeat HR (S810i, Polar Electro, Finland) to analyze HRV. Gas exchange data were measured continuously (ZAN 600 USB, Germany) and data were stored in $5 \mathrm{~s}$ intervals for further analysis. Oxygen uptake $\left(\mathrm{VO}_{2}\right)$, carbon dioxide output (VCO2), pulmonary ventilation (VE) and respiratory exchange ratio (RER) were main variables of interest. Blood samples $(20 \mu \mathrm{l})$ were obtained from a hyperemized earlobe at rest, after warm up, after every single load step and after active and passive recovery for blood lactate (La) and blood glucose (BG) concentration measures (BiosenS_line, EKF Diagnostics, Germany). Gas exchange data and La measures were used to determine the first $\left(\mathrm{VT}_{1} / \mathrm{LTP}_{1}\right)$ and the second $\left(\mathrm{VT}_{2} / \mathrm{LTP}_{2}\right)$ ventilator and lactate turn points as submaximal markers of CRF, respectively [51]. Turn points were determined by means of linear regression break point analysis within defined regions of interest. The first turn point (TP) was calculated between the first workload and 60\% Pmax and the second turn point between the first TP and $\mathrm{P}_{\max }$ [51]. These turn points were shown to represent defined phases of distinct metabolic, hormonal and cardiorespiratory responses [52]. Together maximal oxygen consumption $\left(\mathrm{VO}_{2 \max }\right)$, maximal power output $\left(\mathrm{P}_{\max }\right)$ as well as oxygen uptake and power output at the sub-maximal turn points serve as markers to describe maximal and submaximal CRF. To quantify age dependent exercise performance,the maximal power output (Pmax) from the incremental exercise test was related to the age predicted norm as \%Pmaxpredicted [53] such as $100-120 \%$ of the age predicted norm was suggested as "normal" CRF.

\section{Statistical analyses}

For testing the hypotheses, linear and non-linear regression analyses (quadratic regression)as well as univariate analysis of variance (ANOVA)were conducted. "AL_basic" and "AL_extended" served as the main dependent variables and " $\% \mathrm{P}_{\max }$ predicted" as the independent variable. Post-hoc within-group comparisons were calculated with Bonferroni Post-Hoc tests. A natural logarithmic transformation was applied to the LF, HF, RMSSD and sympathovagal balance (LF/HF) data, as their distribution was skewed. All statistical analysis were performed using the software IBM SPSS Statistics version 21.0 (IBM Corp., NY, USA) and significance level was set at $p<.05$, two-sided for all analyses.

\section{Results}

\section{ALI}

Both ALI_basic $(\mathrm{M}=1.23, \mathrm{SD}=1.32)$ and ALI extended $(M=2.7$, $\mathrm{SD}=2.05)$ signaled mild degrees of burden within this sample. Both AL-indices were strongly interrelated $(r=.753 \mathrm{p}<.001)$.

\section{Cardiorespiratory fitness}

Mean variables for sub-maximal and maximal CRF are shown in Table 2. The mean body mass related oxygen uptake of $41.85 \pm 8.28$ $\mathrm{ml} . \mathrm{kg}^{-1} \cdot \mathrm{min}^{-1}$ (range: 24.2 to $63.5 \mathrm{ml} . \mathrm{kg}^{-1} \cdot \mathrm{min}^{-1}$ ) representing a mean supra-normal CRF level at $124.5 \pm 22.8 \%$ of the age predicted norm level for power output and oxygen uptake [52]. The sub-maximal markers of performance LTP ${ }_{1}$ and LTP $_{2}$ as well as HR and La values were within normal limits for this physically active moderately trained middle aged population.

\begin{tabular}{|l|l|l|l|}
\hline & LTP $_{1}$ & $\mathrm{LTP}_{2}$ & $\mathrm{MAX}$ \\
\hline $\mathrm{HR}\left(\mathrm{min}^{-1}\right)$ & $116.02 \pm 11.21$ & $156.14 \pm 11.10$ & $184.95 \pm 9.60$ \\
\hline $\mathrm{P}(\mathrm{W})$ & $81.45 \pm 29.49$ & $177.24 \pm 37.34$ & $258.28 \pm 47.75$ \\
\hline $\mathrm{VO}_{2}\left(1 . \mathrm{min}^{-1}\right)$ & $1.38 \pm 0.45$ & $2.61 \pm 0.56$ & $3.49 \pm 0.63$ \\
\hline $\mathrm{La}\left(\mathrm{mmol}^{-1}\right)$ & $1.30 \pm 0.37$ & $4.14 \pm 0.88$ & $11.46 \pm 2.02$ \\
\hline
\end{tabular}

Table 2: Variables for sub-maximal and maximal CRF.

Note: Means \pm SD for heart rate (HR), power output $(\mathrm{P})$, oxygen uptake $\left(\mathrm{VO}_{2}\right)$ and blood lactate concentration (La) during incremental cycle ergometer exercise in 89 healthy male office workers (LTP1 - first lactate turn point; LTP2 - second lactate turn point; MAX - maximal work load)

To verify the relationship betweenCRFandALI, a linear regression analysis was computed for both ALI indices, with the variable CRF entered as predictor. Results showed a significant correlation between one variable of $\mathrm{CRF}(\%$ Pmaxpredicted) and ALI_basic $(\beta=-.326, t(84)$ $\left.=-3.12, R^{2}=.11\right)$ as well as \%Pmaxpredictedand ALI_extended $(\beta=$ $\left.-.354, t(84)=-3.42, R^{2}=.13\right)$, suggesting that the higher CRF the lower the allostatic load (Figure 1).

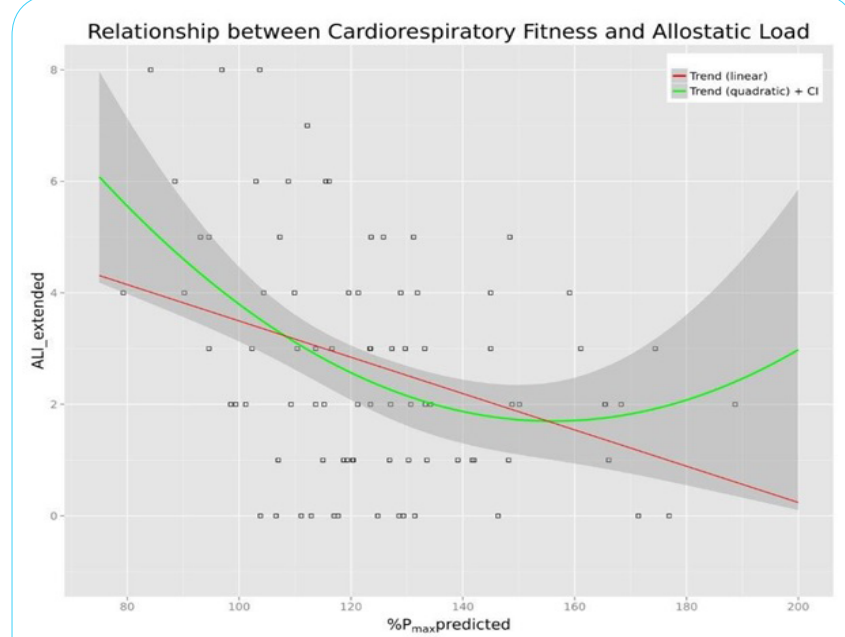

Figure 1: Linear and quadratic regression model for relation of CRF and ALI.

Visual inspection ofFigure 1 raised the impressionthat the relationship between both variables might not be linear.Overly high levels of CRF (i.e., individuals surpas a threshold of CRF> 170\%) could be associated with a slight increase in ALI.Consequently, in a second step a quadratic regression analysis was computed in order to statistically evaluate this finding. Results showed a significantly higher association between ALI and CRF as well as a significant increase in model fit $\left(\beta=-2.255, t(84)=-2.510, R^{2}=.17\right)$. For further analyses the study sample was divided into 5 groups based on \%Pmaxpredicted. Figure 2 shows mean values for ALI.

To verify at which threshold level of CRF significant differences in both ALI-indices can be observed, univariate analysis of variance (ANOVA) was computed. Results showed a significant main effect of group for both, ALI_basic $\left(F[4,79]=4.22, \mathrm{p}=.004, \eta p^{2}=.176\right)$ as well as for ALI_extended $\left(F[4,79]=3.76, \mathrm{p}=.007, \eta p^{2}=.160\right)$. Post-hoc tests (Bonferroni) revealed that there were significant differences in both ALI-indices between group 1 (ALI_basic, $M=2.60, S D=1.43$; ALI_extended, $M=4.70, S D=2.16$ ) and group 3 (ALI_basic, $M=$ $0.79, S D=0.92 ; F[4,79]=21.24, p=<.0001, \eta p^{2}=.371$; ALI_extended, 
Citation: Paletta A, Suchar G, Hübler T, Juschitz D, Mayer K, et al. (2017) Time Experience of Burnout Patients: A Qualitative Diary Analysis. Int J Phys Ther Rehab 3: 126. doi: https://doi.org/10.15344/2455-7498/2017/126

Page 6 of 7

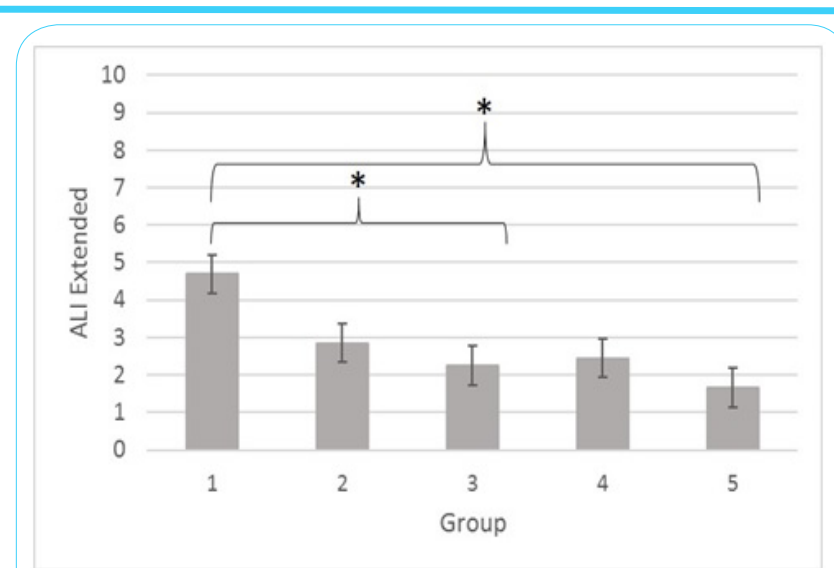

Figure 2: Mean values for ALI_extended and $\% \mathrm{P}_{\max }$ predicted.

Note: Group $1<100 \%$ Pmaxpredicted (80 - 100\%), group 2 people within normal range $\left(100-120 \% \mathrm{P}_{\max }\right.$ predicted) and groups 3 (120 - 140\%Pmaxpredicted), 4 (140 - $160 \% \mathrm{P}_{\max }$ predicted) and 5 (160 $180 \% \mathrm{P}_{\max }$ predicted) people above normal range of CRF. ${ }^{\star} p<.05$.

$\left.M=2.25, S D=1.56 ; F[4,79]=14.83, p=<.0001, \eta p^{2}=.292\right)$ and also between group 1 and group 5 (ALI_basic, $M=0.89, S D=0.78 ; \mathrm{F}[4,79]$ $=10.12, p=.005, \eta p^{2}=.373$; ALI_extended $M=1.67, S D=1.12 ; F[4$, $\left.79]=14.22, p=.002, \eta p^{2}=.456\right)$ with comparably large effect sizes. These results suggest that individuals with lower exercise performance than normal $(<100 \%$ Pmaxpredicted $)$ showed higher stress related signs of physiological burden and that only above a threshold level of $120 \%$ CRF a significant reduction of the psychophysiological signs of burden could be observed.

Additional analyses were computedto explore associations between $\% \mathrm{P}_{\max }$ predicted and each of the physiological variables of the ALI separately. There were significant correlations with WHR $(r=-.271$, $p=<.013)$, HR $(r=-.555, p=<.001)$, DBP $(r=-.279, p=<.013)$ $\operatorname{SDNN}(r=.302, p=<.005)$ as well as with $\operatorname{rMSSD}(r=.214, p=<.05)$.
These results suggest that higher CRF was associated with lower WHR, HR, DBP, and higher SDNN and rMSSD. Additional analyses were also computed for alternative quantifications of CRF like $\mathrm{VO}_{2 \max }$, LTP $_{1}$, LTP $_{2}$ and $\mathrm{P}_{\max }$. Results are shown in Table 3.

\section{Self-rated work stress}

In order to verify the working stress conditions of the individuals, a ratio between effort and reward was calculated. A mean value of 1.25 ( $\mathrm{SD}=0.38$ ) confirmed that the sample showed higher effort than reward ratings, indicating stress. To find out, whether there was a correlation between the subscales of the ERI and both ALIindices, additionallinear regression analysis were computed.For both analyses, subtests of ERI were used as predictor variables. Results ( Table 4 and Table 5) showed a significant correlation between the subscale overcommitment and ALI_basic $(\beta=.214, t(84)=2.004$,

\begin{tabular}{|l|l|l|l|}
\hline Variable & B & SE B & $\beta$ \\
\hline ALI basic $x$ over commitment & .12 & .06 & $.21^{*}$ \\
\hline ALI extended x ratio effort/reward & 1.14 & .58 & $.21^{*}$ \\
\hline
\end{tabular}

Table 4: Linear regression analyses between AL and ERI subtests $(n=86)$.

Note. $\mathrm{R}^{2}=.05\left({ }^{*} p<.05\right)$ for over commitment; $\mathrm{R}^{2}=.04\left({ }^{*} p<.05\right)$ for ratio effort/ reward.

\begin{tabular}{|l|l|l|l|}
\hline Variable & $B$ & $S E B$ & $\beta$ \\
\hline$\% \mathrm{P}_{\max }$ predictedx overcommitment & .04 & .02 & $.27^{\star}$ \\
\hline$\% \mathrm{P}_{\max }$ predictedx ratio effort/reward & -.00 & .00 & $-.25^{\star}$ \\
\hline
\end{tabular}

Table 5: Linear regression analyses between \%Pmaxpredicted and ERI subtests $(n=86)$ Note.

$\mathrm{R}^{2}=.07\left({ }^{*} p<.05\right)$ for reward; $\mathrm{R}^{2}=.06(\mathrm{~T} p<.10)$ for ratio effort $/$ reward.

$R^{2}=.046$ ), but not for the subscales effort and reward, suggesting that higher overcommitmentwas associated with higher allostatic load. A rather moderatecorrelation could be found between the ratio effort/ reward and ALI_extended $\left(\beta=.208, t(84)=1.952, R^{2}=.032\right)$, but not

\begin{tabular}{|c|c|c|c|c|c|c|c|}
\hline & $\mathrm{VO}_{2}$ & $\mathrm{LTP}_{1}$ & $\mathrm{LTP}_{2}$ & $\% \mathrm{P}_{\max }$ predicted & $\mathrm{P}_{\max }$ & Mean & SD \\
\hline Effort & -.12 & .04 & -.06 & -.15 & -.10 & 10.49 & 1.40 \\
\hline Reward & .15 & $.22^{*}$ & $.25^{*}$ & $.27^{*}$ & $.28^{*}$ & 19.45 & 3.57 \\
\hline Overcommitment & -.14 & -.08 & -.17 & -.17 & -.18 & 15.01 & 2.36 \\
\hline Efford-Reward-Ratio & -.17 & -.13 & -.20 & $-.25^{*}$ & $-.23^{*}$ & 1.25 & .38 \\
\hline ALI Basic & $-.37^{*}$ & -.16 & $-.22^{*}$ & $-.33^{*}$ & $-.22^{*}$ & 1.23 & 1.32 \\
\hline ALI Extended & $-.44^{*}$ & $-.25^{*}$ & $-.27^{*}$ & $-.35^{*}$ & $-.27^{\star}$ & 2.70 & 2.05 \\
\hline HR 24h (BPM) & $-.42^{*}$ & $-.39^{*}$ & $-.46^{*}$ & $-.55^{*}$ & $-.46^{*}$ & 69.36 & 8.20 \\
\hline SDNN 24h (ms) & $.27^{*}$ & $.26^{*}$ & $.32^{*}$ & $.29^{*}$ & $.31^{*}$ & 93.60 & 23.34 \\
\hline rMSSD 24h (ms) & $.29^{*}$ & $.23^{*}$ & $.22^{*}$ & .21 & .24 & 3.50 & .37 \\
\hline $\mathrm{LF} / \mathrm{HF}\left(\mathrm{ms}^{2}\right)$ & -.14 & -.11 & -.08 & -.15 & .23 & .75 & .19 \\
\hline SBP (mmHg) & -.19 & -.12 & -.16 & -.13 & -.17 & 120.98 & 10.19 \\
\hline $\mathrm{DBP}(\mathrm{mmHg})$ & $-.30^{*}$ & -.28 & $-.33^{*}$ & $-.27^{*}$ & $-.35^{*}$ & 79.24 & 8.06 \\
\hline WHR & $-.38^{*}$ & $-.27^{*}$ & $-.24^{*}$ & $-.27^{*}$ & $-.22^{*}$ & .92 & .10 \\
\hline Epinephrine $(\mu \mathrm{g} / 24 \mathrm{~h})$ & -.11 & -.06 & -.13 & -.13 & -.11 & 7.79 & 3.28 \\
\hline Norepinephrine $\mu \mathrm{g} / 24 \mathrm{~h}$ ) & -.16 & .03 & -.09 & -.11 & -.11 & 45.31 & 13.24 \\
\hline Cortisol-Increase (\% t0-t30) & -.01 & .11 & .04 & -.05 & .17 & 64.11 & 84.15 \\
\hline
\end{tabular}

Table 3: Pearson correlation coefficients and descriptive statistics (Mean, SD) of ALI parameters and parameters of CRF ( $\mathrm{n}=86$ ).

Note: ${ }^{*}=$ significant $\mathrm{p}<.05$ 
Citation: Paletta A, Suchar G, Hübler T, Juschitz D, Mayer K, et al. (2017) Time Experience of Burnout Patients: A Qualitative Diary Analysis. Int J Phys Ther Rehab 3: 126. doi: https://doi.org/10.15344/2455-7498/2017/126

Page 7 of 7

for the subscales effort and reward separately. These results suggest that the higher the experienced work stress the higher allostatic load was. Additional correlations were computed to explore associations between ERI and each of the physiological variables of the ALI separately.We decided on a Bonferroni alpha adjustment to $p<.016$ to overcome the problem of alpha inflation. Results showed significant correlations between the ratio of effort/reward and SDNN $(r=$ $-.281, p=<.009)$ as well as effort/reward and rMSSD $(r=-.275$, $\mathrm{p}=<.011$ ), suggesting that higher experienced working stress was accompanied by lower SDNN and the lower parasympathetic activity. Significant correlations could also be found for reward and SDNN ( $\mathrm{r}$ $=.278, p=<.010)$ and $\operatorname{rMSSD}(r=.294, p=<.006)$, suggesting thata higher experienced reward was accompanied with higher HRV. No significant correlationswith any physiological variablewere found for effort and over commitment. Additional analyses were conducted to verify possible correlations between subtests of ERI and parameter of CRF. To verify the association between $\% \mathrm{P}_{\max }$ predicted and ERI, an additional linear regression analysis was computed. Results showed a significant correlation between \%Pmaxpredicted and reward $(\beta=$ $\left..271, t(84)=2.552, R^{2}=.074\right)$ as well as between $\% \mathrm{P}_{\max }$ predicted and effort/reward ratio $\left(\beta=-.253, t(84)=-2.36, R^{2}=.064\right)$, suggesting that perceived reward was significantly higher with higher CRF. Additionally, results showed significantly higher effort/reward imbalance the lower CRF. No correlations could be found between \%Pmaxpredicted and effort and over commitment, respectively. Additional analyses were also computed for subtests of the ERI and alternative quantifications of CRF like $\mathrm{VO}_{2 \max }, \mathrm{LTP}_{1}$, LTP $\mathrm{L}_{2}$ and Pmax. Results do not suggest any additional effect.

\section{Discussion}

The objective of the present study was to investigate whether CRFis associated with physiological signs of burden, which could be seen as a consequence of stressful working conditions. Therefore, individuals working under stressful working conditions were analyzed by measuring CRF, self-reported work stress, stress related variables of the SAM (catecholamines, HRV and blood pressure) and the HPA axis (cortisol). In order to capture the amount of dysregulations multiple physiological variables were integrated into two ALIs[54]. Because previous studies almost exclusively focused on the negative consequences of stressful working conditions and self-reported stress/ burnout symptoms on physiological dysregulations, the aim of this study was to explore the relation between available positive resources like CRF and cumulative physiological burden. Therefore, we analyzed different objective markers of physical fitness.

\section{ALI}

In line with expectations, both the ALI basic and the extended ALI score were significantly lower with increasing CRF (specifically, $\% \mathrm{P}_{\max }$ predicted). In particular, individuals with higher CRF showed significantly lower WHR, HR, DBP and significantly higher rMSSD compared to individuals with lower CRF. These results suggest that lower physical fitness might be accompanied by higher signs of physiological burden. This finding corroborates previous studies suggestinga buffering effect of physical exercise/activity on HR, HRV, blood pressure, and WHR. A current review on HRV, physical activity and working stress could show a strong association between workplace stress and reduced HRV in employees [55]. Several studies $[16,56,57]$ reported a reduced HRV in employeesreporting work related stress. The authors concluded that assessing the autonomic functioning could be a straight forward and effective way to identify work stress. It is thus surprising that HRV has not yet been routinely integrated in the AL metric.

It is notable that all included studies in this review assessed the level of physical activity or exercise with self-reports in a descriptive function. The authors encouraged more studies concerning physical activity and exercise intervention for better understanding the benefits of physical activity on both stress and HRV but neglected the possible influence of CRF on the signs of physiological burden resulting from stressful working conditions. Interestingly, in this context it has never been discussed whether the actual status ofCRF should be integrated as a fixed component in psychophysiological stress diagnosesalongside the evaluation of stress related variables of the SAM and HPA axis.The current study suggests that this could be a promising addendum in order to better evaluate individual physiological processes under stress. Moreover, the hypothesis that CRF could counteract physiological dysregulations could prove fruitful for designing intervention programs.Moreover, the findings of this study may help answer the question whysome individuals under same working conditions are affected by stress related symptomswhile others are not. Currently, positive resources are not sufficiently valued when diagnosing the impact of work stress on physiology.Moreover, the findings of this study could help to clarify the emerging inconsistency in the field ofstress/burnout and psychophysiology[58].It is well known that available resources could have a buffering effect on physiological stress responses and health[59]. Several studies related work stress and burnout symptoms to $\mathrm{AL}[46,60]$ and reaffirmed significant correlations between chronic stress and higher AL. Although the total score of both AL quantifications (basic and extended, respectively) was quite low in this study, the most sensitive measures seemed to be those of the SAM axis and not those of the HPA axis.Of note, parasympathetic -mediated HRV (rMSSD) was negatively associated with work stress, which corroboratesother research showing high correlations between stress and decreased rMSSD[61]. Previous findings suggesting positive associationsbetween HRV and CRF[37] could also be replicated in the present study.Individuals with higher CRF showed significantly higher rMSSD compared to individuals with lower CRF.

One explanation why no significant associations were found for epinephrine and norepinephrine could be that catecholamines are short acting and short living molecules, thus making them more sensitive toacute stress[7]. Hence, an aggregate level across 24 hours could have been too coarse and biased by other, momentary circumstances.

\section{Cardiorespiratory fitness}

In line with expectations, CRF was significantly higher in individuals who showed lower signs of physiological burden.Results also showed significant correlations between CRF and WHR, diastolic blood pressure, SDNN and rMSSD. Specifically, a higher CRF was associated with lower WHR, diastolic blood pressure and higher HRV (in particular, indices of parasympathetic activity). These results are compatible with research suggesting that physical activity and exercise could ameliorate stress related disorders and signs of burnout [55]. It is assumed that physical activity results in higher CRF,which in turn could promote resilience to mental stress[62]. Positive consequences of physical activity asan intervention in the workplace have been found in previous studies[63], suggesting that physical activity could constitute an effective tool for buffering allostatic load. This topic was also examined from another perspective. It has been questioned 
Citation: Paletta A, Suchar G, Hübler T, Juschitz D, Mayer K, et al. (2017) Time Experience of Burnout Patients: A Qualitative Diary Analysis. Int J Phys Ther Rehab 3: 126. doi: https://doi.org/10.15344/2455-7498/2017/126

Page 6 of 7

whether individual coping programs can counteract workplace stressors and are powerful enough to eliminate the cause of stressrelated symptoms and burnout[64]. Such programs regard the risk for stress exclusively as a result of sustained high demands stemming from the workplace environment. Certainly, individual interventions cannot influence or change the working environment but they could influence physiological adaptation processes and potentially strengthen available resources in a positive way[63]. Hence, it might be asked why the evaluation of available individual resources like CRFis not a fixed component of stress evaluation? It should be noted though that the presumed positive relationship between higher stress resilience and higher physical fitness has to be demonstrated yet [62] One possible reason for the heterogeneity of findings could be related to different methodological approaches in the assessment of physical fitness. Results of a review concerning this topic reported that all 18 studies included in this review assessed the level of physical activity or exercise exclusively with self-report data[55], thus questioning the validity of the assessment. Moreoverit seems challenging to specify a specific CRF threshold that should be met in order to verify positive effects on stress-related psychophysiologicalsystems.

To gain more clarity about the dose-response relationship,our analysis suggested that participants had to reach $120-140 \%$ of agerelated CRF to achieve this positive effect on AL. This effect is likely to shrink the higher the percentage of CRF. It is interesting to note that comparably high levels of CRF tended to be accompanied by increased $\mathrm{AL}$, as became evident by a curvilinear regression. Of note, this finding is compatible with research suggesting that overtraining could be associated with physiological dysregulations [67]. It should be emphasized though that in order to substantiate this finding, further research with individuals scoring over a wider range of CRF is certainly warranted.

Importantly, the worst health-related effect had those, who showed CRFvalues lower than 100\%Pmaxpredicted.Interestingly, a cut-off point of approximately 10-12 METs (metabolic equivalent to CRF), which is comparable with the average of oxygen intake in our sample size, was shown to be related to several health risk factors, and the highest mortality risk was in the $<10$ METs / $<30 \mathrm{~kg} / \mathrm{m} 2$ subgroup [65]. These authors argued that given the comparatively limited impact of BMI, more emphasis should be placed on measuring exercise capacity and developing strategies for its improvement in cardiovascular disease prevention programs. Similar cut-off scores for CRF have been documented for the relationship between CRF and diabetes risk. After adjustment, patients achieving $\geq 12$ METs had a $54 \%$ lower risk of incident diabetes compared with patients achieving $<6$ METs [66].

\section{ERI}

Assessment of work stress was performed with the ERI [12], because this inventory is widely used in stress research to identify work related stress[41]. Significant correlations could be found between the subscale reward and the effort/reward ratio with variables of the SAM axis (in particular, DBP, SDNN and rMSSD), but not with HPA axis (cortisol). This indicates that an imbalance between high effort and low reward could increase the risk of negative effects on health, especially on variables of the SAM axis.These findings are in agreement with results of prospective and cross-sectional studies, indicating health-adverse effects of an imbalance between perceived effort and received reward [41]. Results of a review study concerning effort/reward imbalance related high effort and low reward to poor employee health[68]. They also mentioned that the combination of high effort and low reward could enableidentification of employees at risk for an early stage of cardiovascular disease. With respect to the present sample, the possible risk of reduced health resulting from this imbalance could be shown by decreased parasympathetic activity, higher DBP and lower SDNN. This is consistent with subjective evaluations from participants, attributing working stress not to their expended effort but rather to non-received reward in terms of money, esteem and job opportunities including job security. In line with this reasoning,a higher degree of appreciation towards employees, especially within different hierarchical levels, has been found to decrease the experienced stress/burnout-symptoms significantly[69-71]. Findings concerning the significant correlation between the subscale over-commitment and AL corroborate other research, stating that high over-commitment leads to impaired health [68]. Of note, it is hypothesized that a high imbalance between effort and reward could lead to negative health outcomes as a consequence of a compromised health behavior. This could possibly explain the significant correlation between \%Pmaxpredicted and reward respectively effort/reward ratio.

\section{Limitations}

These findings should be considered in the context of several limitations. Firstly, the cross-sectional design gives no clear information about causality of associations. Hence, it remains to be explored whether higher CRF could influence AL or vice versa. Probably bidirectional effects are prevalent. Secondly, although we assessed the hours per week spent with physical activity, we do not have additional information concerning the type and intensity of physical activity, which could complement the findings. Thirdly, it should be noted that the study sample was quite homogeneous with respect to education, age, CRF, and perceived work stress. Further studies are needed to analyze the interrelation between CRF, psychological and physiological stress-related processes in more heterogeneous or more compromised samples. Fourthly, the findings on effort, reward, and over-commitment need to be interpreted with cation, because of rather low reliability of the subscales of the ERI. Finally, the study analyzed men only. Hence, the generalizability to women needs to be explored in future studies.

\section{Conclusion}

In summary, the results of the present study suggest a relationship between work-related stress, psychophysiological signs of burden and CRF. However, the extent of the positive benefits of CRF on AL remains to be elucidated. Thus, future research, especially studies with heterogeneous samples concerning different jobs, different working stress conditions, different educational levels as well as different CRF levelsis needed to better understand how different levels of CRF can minimize psychophysiological dysregulation.Examination of the results reveals that correlations between CRF and AL were only found within variables of the SAM axis (except catecholamines) but not within variables of the HPA axis. This could indicate that variables of the SAM axis, especially blood pressure and rMSSD seems to be particularly sensitivefor evaluating first signs of stress related physiological burden.

\section{Competing Interests}

The authors declare that they have no competing interests. 
Citation: Paletta A, Suchar G, Hübler T, Juschitz D, Mayer K, et al. (2017) Time Experience of Burnout Patients: A Qualitative Diary Analysis. Int J Phys Ther Rehab 3: 126. doi: https://doi.org/10.15344/2455-7498/2017/126

Page 7 of 7

\section{Ethical Approval}

All procedures performed in studies involving human participants were in accordance with the ethical standards of the institutional and/ or national research committee (GZ. 39/7/63 ex 2013/14) and with the 1964 Helsinki declaration and its later amendments or comparable ethical standards.

\section{Acknowledgements}

This study received founding in equal parts by the Austrian Social Insurance for Occupational Risks (AUVA) and the Austrian Pension Insurance Institution (PVA). The contribution of Bernhard Weber creating the graph is greatly acknowledged.

\section{References}

1. Rowe DS (2012) The stress burden: strategies for management. Nevada RNformation: 12

2. Nash K (2013) The growth of burnout syndrome. Charter: 34

3. Chrousos GP, Gold PW (1992) The concepts of stress and stress system disorders: Overview of physical and behavioral homeostasis. JAMA 267 1244-1252.

4. Schneiderman N, Ironson G, Siegel SD (2005) Stress and Health Psychological, Behavioral, and Biological Determinants. Annual Review of Clinical Psychology 1: 607-628.

5. Selye H (1978) The stress of life. (Rev. ed.). McGraw-Hill paperbacks, McGraw-Hill, New York.

6. Chrousos GP, Loriaux L, Gold PW (1988) Mechanisms of physical and emotional stress. Advances in experimental medicine and biology, Volume 245, Springer Science+Business Media, LLC, New York.

7. Chandola T, Heraclides A, Kumari M (2010) Psychophysiological biomarkers of workplace stressors. Neuroscience \&Biobehavioral Reviews 35: 51-57.

8. Shirom A, Melamed S, Toker S (2005) Burnout and health review. Current knowledge and future research direction. In: Hodgkinson JP, Ford JK (Ed). International Review of Industrial and Organizational Psychology, vol 20. John Wiley \& Sons, Chichester.

9. Ahola K, Honkonen T, Isometsä E, Kalimo R, Nykyri E, et al. (2005) The relationship between job-related burnout and depressive disordersresults from the Finnish Health 2000 Study. Journal of Affective Disorders 88: $55-62$.

10. Honkonen T, Ahola K, Pertovaara M, Isometsä E, Kalimo R, et al. (2006) The association between burnout and physical illness in the general populationresults from the Finnish Health 2000 Study. Journal of Psychosomatic Research 61: 59-66.

11. Suls J, Bunde J (2005) Anger, anxiety, and depression as risk factors for cardiovascular disease: the problems and implications of overlapping affective dispositions. Psychological Bulletin 131: 260-300.

12. Siegrist J, Siegrist K, Weber I (1986) Sociological concepts in the etiology of chronic disease: the case of ischemic heart disease. Social Science \& Medicine (1982) 22: 247-253.

13. Siegrist $J$ (1998) Reciprocity in basic social exchange and health: can we reconcile person-based with population-based psychosomatic research? Journal of Psychosomatic Research 45: 99-105.

14. Kivimaki M, Virtanen M, Elovainio M, Kouvonen A, Vaananen A, et al. (2006) Work stress in the etiology of coronary heart disease-a meta-analysis Scandinavian Journal of Work, Environment \& Health 32: 431-442.

15. Alexandrova-Karamanova A, Todorova I, Montgomery A, Panagopoulou E, Costa P, et al. (2016) Burnout and health behaviors in health professionals from seven European countries. International Archives of Occupational and Environmental Health. Int Arch Occup Environ Health 89: 1059-1075.

16. Chandola T, Britton A, Brunner E, Hemingway H, Malik M, et al. (2008) Work stress and coronary heart disease: what are the mechanisms? European Heart Journal 29: 640-648.
17. Danhof-Pont MB, van Veen T, Zitman FG (2011) Biomarkers in burnout: A systematic review. Journal of Psychosomatic Research 70: 505-524.

18. Sapolsky RM, Lewis CK, McEwen BS (2002) The Neuroendocrinology of Stress and Aging: The Glucocorticoid Cascade Hypothesis. Science of Aging Knowledge Environment Endocr Rev 7: 284-301

19. Kirschbaum C, Hellhammer DH (1989) Salivary cortisol in psychobiological research: an overview. Neuropsychobiology 22: 150-169.

20. McEwen BS (1993) Stress and the individual. Mechanisms leading to disease. Archives of Internal Medicine 153: 2093-2101.

21. Sterlin P, Eyer J (1988) Allostasis: A new paradigm to explain arousal pathology. In: Fisher S, Reason JT (Ed). Handbook of life stress, cognition, and health. Wiley, Chichester, New York, pp 629-649.

22. Lacey JI, Lacey BC (1958) Verification and Extension of the Principle of Autonomic Response-Stereotypy. The American Journal of Psychology 71: 50

23. Karlamangla AS, Singer BH, McEwen BS, Rowe JW, Seeman TE (2002) Allostatic load as a predictor of functional decline. Journal of Clinical Epidemiology 55: 696-710.

24. Gay JL, Salinas JJ, Buchner DM, Mirza S, Kohl HW3, et al. (2015) Meeting physical activity guidelines is associated with lower allostatic load and inflammation in Mexican Americans. Journal of immigrant and minority health / Center for Minority Public Health 17: 574-581.

25. Tsatsoulis A, Fountoulakis S (2006) The protective role of exercise on stress system dysregulation and comorbidities. Annals of the New York Academy of Sciences 1083: 196-213.

26. Rimmele U, Seiler R, Marti B, Wirtz PH, Ehlert U, et al. (2009) The level of physical activity affects adrenal and cardiovascular reactivity to psychosocial stress. Psychoneuroendocrino 34: 190-198.

27. Fleshner M (2005) Physical activity and stress resistance: sympathetic nervous system adaptations prevent stress-induced immunosuppression. Exercise and sport sciences reviews 33: 120-126.

28. Caspersen CJ, Powell KE, Christenson GM (1985) Physical activity, exercise, and physical fitness: definitions and distinctions for health-related research. Public Health Reports (Washington, D.C. : 1974) 100: 126-131.

29. Åberg MAI, Waern M, Nyberg J, Pedersen NL, Bergh Y, et al. (2012) Cardiovascular fitness in males at age 18 and risk of serious depression in adulthood: Swedish prospective population-based study. The British Journal of Psychiatry: The Journal of Mental Science 201: 352-359.

30. Boettger S, Wetzig F, Puta C, Donath L, Müller H, et al. (2009) Physical fitness and heart rate recovery are decreased in major depressive disorder. Psychosomatic Medicine 71: 519-523.

31. Sui X, Laditka JN, Church TS, Hardin JW, Chase N, et al. (2009) Prospective study of cardiorespiratory fitness and depressive symptoms in women and men. Journal of Psychiatric Research 43: 546-552.

32. Wamala SP, Mittleman MA, Horsten M, Schenck-Gustafsson K, OrthGomér K (2000) Job stress and the occupational gradient in coronary heart disease risk in women. The Stockholm Female Coronary Risk Study.Social Science \& Medicine (1982) 51: 481-489.

33. Forcier K, Stroud LR, Papandonatos GD, Hitsman B, Reiches M, et al (2006) Links between physical fitness and cardiovascular reactivity and recovery to psychological stressors: A meta-analysis. Health Psychology 25: 723-739.

34. Gerber M, Brand S, Elliot C, Holsboer-Trachsler E, Puhse U, et al. (2013) Aerobic exercise training and burnout: a pilot study with male participants suffering from burnout. BMC Research Notes 6: 78.

35. Bretland RJ, Thorsteinsson EB (2015) Reducing workplace burnout: the relative benefits of cardiovascular and resistance exercise. Peer Journal 3: e891.

36. Ben-Ari ET (2000) BioBriefs. BioScience 50: 96.

37. Teisala T, Mutikainen S, Tolvanen A, Rottensteiner M, Leskinen T, et al (2014) Associations of physical activity, fitness, and body composition with heart rate variability-based indicators of stress and recovery on workdays: a cross-sectional study. Journal of Occupational Medicine and Toxicology 9: 16.

38. Williams PT (2001) Physical fitness and activity as separate heart disease risk factors: a meta-analysis. Med Sci Sports Exerc 33:754-61. 
Citation: Paletta A, Suchar G, Hübler T, Juschitz D, Mayer K, et al. (2017) Time Experience of Burnout Patients: A Qualitative Diary Analysis. Int J Phys Ther Rehab 3: 126. doi: https://doi.org/10.15344/2455-7498/2017/126

Page 7 of 7

39. Lee D, Artero EG, Sui X, Blair SN (2010) Mortality trends in the general population: the importance of cardiorespiratory fitness. Journal of Psychopharmacology 24: 27-35.

40. Margraf J (1994) Mini-DIPS: Diagnostisches Kurz-Interview bei psychischen Störungen, Springer Berlin Heidelberg; Imprint; Springer, Berlin, Heidelberg

41. Siegrist J, Starke D, Chandola T, Godin I, Marmot M, et al. (2004) The measurement of effort-reward imbalance at work: European comparisons. Social Science \& Medicine 58: 1483-1499.

42. Tonello L, Reichert FF, Oliveira-Silva I, Del Rosso S, LEICHT AS, et al. (2016) Correlates of Heart Rate Measures with Incidental Physical Activity and Cardiorespiratory Fitness in Overweight Female Workers Front Physiol 6. 24 .

43. Traunmüller C, Gaisbachgrabner K, Lackner H, Schwerdtfeger A (2016) Burnout of the mind - burnout of the body? Journal of Psychophysiology (In Press)

44. Seeman TE, McEwen BS, Rowe JW, Singer BH (2001) Allostatic load as a marker of cumulative biological risk: MacArthur studies of successful aging. Proceedings of the National Academy of Sciences 98: 4770-4775

45. Pruessner JC, Wolf OT, Hellhammer DH, Buske-Kirschbaum A, von Aue K, et al. (1997) Free cortisol levels after awakening: A reliable biologica marker for the assessment of adrenocortical activity. Life Sci 61: 25392549 .

46. Bellingrath S, Weigl T, Kudielka BM (2009) Chronic work stress and exhaustion is associated with higher allostastic load in female school teachers. Stress (Amsterdam, Netherlands) 12: 37-48.

47. Stalder T, Kirschbaum C, Kudielka BM, Adam EK, Pruessner JC, et al. (2016) Assessment of the cortisol awakening response: Expert consensus guidelines. Psychoneuroendocrinology 63: 414-432.

48. Task Force of the European Society of Cardiology and the North American Society of Pacing and Electrophysiology (1996) Heart rate variability: Standards of measurement, physiological interpretation and clinical use. Circulation 93: 1043-1065.

49. Delaney JP, Brodie DA (2000) Effects of short-term psychological stress on the time and frequency domains of heart-rate variability. Perceptual and motor skills 91 : $515-524$

50. Malliani A, Pagani M, Lombardi F, Cerutti S (1991) Cardiovascular neura regulation explored in the frequency domain. Circulation 84: 482-492.

51. Hofmann P, Tschakert G (2011) Special needs to prescribe exercise intensity for scientific studies. Cardiology Research and Practice 2011 $1-10$.

52. Moser O, Tschakert G, Mueller A, Groeschl W, Pieber TR, et al. (2015) Effects of High-Intensity Interval Exercise versus Moderate Continuous Exercise on Glucose Homeostasis and Hormone Response in Patients with Type 1 Diabetes Mellitus Using Novel Ultra-Long-Acting Insulin. PLoS ONE 10: e0136489.

53. Wonisch M, Berent R, Klicpera M, Laimer H, Marko C, et al. (2008) Practice guidelines for exercise testing. Austrian Journal of Cardiology 15(Suppl A), 3-17. (In German: English abstract)

54. Karlamangla AS, Singer BH, Seeman TE (2006) Reduction in allostatic load in older adults is associated with lower all-cause mortality risk: MacArthur Studies of Successful Aging. Psychosom Med 68: 500-507.

55. Tonello L, Rodrigues FB, Souza JWS, Campbell CSG, LEICHT AS, et al (2014) The role of physical activity and heart rate variability for the control of work related stress. Frontiers in Physiology 5: 67.

56. Clays E, Bacquer D de, Crasset V, Kittel F, Smet P de, et al. (2011) The perception of work stressors is related to reduced parasympathetic activity. International Archives of Occupational and Environmental Health 84: 185 191.

57. Uusitalo A, Mets T, Martinmaki K, Mauno S, Kinnunen U, et al. (2011) Heart rate variability related to effort at work. Applied Ergonomics 42: 830-838.

58. Mauss D, Li J, Schmidt B, Angerer P, Jarczok MN (2015) Measuring allostatic load in the workforce: a systematic review. Industrial health 53 : 5-20.

59. Cohen S, Wills TA (1985) Stress, Social Support, and the Buffering Hypothesis. Psychol Bull 98: 310-357.
60. Juster R, Sindi S, Marin M, Perna A, Hashemi A, et al. (2011) A clinical allostatic load index is associated with burnout symptoms and hypocortisolemic profiles in healthy workers. Psychoneuroendocrino 36: 797-805.

61. Orsila R, Virtanen M, Luukkaala T, Tarvainen M, Karjalainen P, et al. (2008) Perceived mental stress and reactions in heart rate variability-a pilot study among employees of an electronics company. Int J Occup Saf Ergon 14: 275-283.

62. Hamer M (2012) Psychosocial stress and cardiovascular disease risk: the role of physical activity. Psychosomatic Medicine 74: 896-903.

63. Juster R, McEwen BS, Lupien SJ (2010) Allostatic load biomarkers of chronic stress and impact on health and cognition. Neuroscience \& Biobehavioral Reviews 35: 2-16.

64. Halbesleben JRB (2006) Action Research as a Burnout Intervention: Reducing Burnout in the Federal Fire Service. The Journal of Applied Behavioral Science 42: 244-266.

65. McAuley PA, Blaha MJ, Keteyian SJ, Brawner CA, Al Rifai M, et al. (2016) Fitness, Fatness, and Mortality: The FIT (Henry Ford Exercise Testing) Project. Am J Med 129: 960-965.

66. Juraschek SP, Blaha MJ, Blumenthal RS, Brawner C, Qureshi W, et al. (2015) Cardiorespiratory fitness and incident diabetes: the FIT (Henry Ford Exerclse Testing) project. Diabetes Care 38: 1075-1081.

67. Brooks K, Carter J.: Overtraining, Exercise, and Adrenal Insufficiency. J Nov Physiother 16: 11717

68. van Vegchel N, Jonge J de, Bosma H, Schaufeli W (2005) Reviewing the effort-reward imbalance model: drawing up the balance of 45 empirical studies. Social Science \& Medicine (1982) 60: 1117-1131.

69. Etzion D (1984) Moderating effect of social support on the stress-burnout relationship. Journal of Applied Psychology 69: 615-622.

70. Bakker AB, Demerouti E, Euwema MC (2005) Job resources buffer the impact of job demands on burnout. Journal of occupational health psychology 10: 170-180.

71. Cordes CL, Dougherty TW (1993) A Review and aniintegration of Research on Job Burnout. Academy of Management Review 18: 621-656. 